\title{
Arquitectura empresarial: estrategia para un cambio organizacional apoyado en la gestión de tecnologias de la información. Una revisión bibliográfica
}

Daniel Felipe Giraldo-Ladino ${ }^{1}$

Universidad Nacional Abierta y a Distancia - UNAD danielpipe03@gmail.com

Ángela Dayan Garay-Villada²

Universidad Nacional Abierta y a Distancia - UNAD andagavi@gmail.com

Diana Marcela Cardona-Román ${ }^{3}$

Universidad Nacional Abierta y a Distancia - UNAD

diana.cardona@unad.edu.co

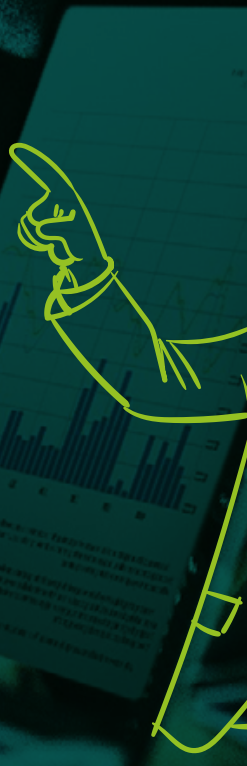

DOI: https://doi.org/10.21158/23823399.v8.n0.2020.2613

Fecha de recepción: 12 de marzo de 2020

Fecha de aprobación: 08 de junio de 2020

Cómo citar este artículo: Giraldo-Ladino, D. F.; Garay-Villada, A. D.; Cardona-Román, D. M. (2020). Arquitectura empresarial: estrategia para un cambio organizacional apoyado en la gestión de tecnologías de la información. Una revisión bibliográfica. Revista Ontare, 8, 49-70.

DOI: https://doi.org/10.21158/23823399.v8.n0.2020.2613

\footnotetext{
${ }^{1}$ Ingeniero electrónico de la Universidad Nacional Abierta y a Distancia - UNAD. Especialista en Aplicación de TIC para la Enseñanza, de la Universidad De Santander - Campus Bucaramanga. Magister en Gestión de Tecnología de Información de la Universidad Nacional Abierta y a Distancia - UNAD. ORCID: https://orcid.org/0000-0001-9004-0943

${ }^{2}$ Ingeniera de Sistemas de la Universidad Nacional Abierta y a Distancia - UNAD. Especialista en auditoría y control fiscal de la Universidad Antonio Nariño - Sede Bogotá. Estudiante de la maestría en Gestión de Tecnologías de Información de la Universidad Nacional Abierta y Distancia - UNAD. ORCID: https://orcid.org/0000-0001-7989-5529

${ }^{3}$ Ingeniera de sistemas de la Universidad de los Llanos. Especialista en Entornos Virtuales de Aprendizaje del Centro de altos estudios universitarios OEI. Magister en Ingeniería de Sistemas y Computación y doctora Ingeniería - Industria y Organizaciones por la Universidad Nacional de Colombia. ORCID: https://orcid.org/0000-0003-0953-5178
} 



\section{RESUMEN}

El objetivo principal de este artículo es realizar una revisión bibliográfica acerca de la arquitectura empresarial (AE), la gestión estratégica de tecnologías de la información ( $\mathrm{TI}$ ) y el cambio organizacional, presentando su definición y su asociación con el fin de afrontar el cambio en las organizaciones. De acuerdo con diferentes estudios en los que se menciona la AE como un enfoque ágil, se identifican los principales componentes de la organización y su relación en el propósito de obtener los objetivos del negocio. En la actualidad, varias empresas se apoyan en diferentes estrategias debido a su complejidad y, por tanto, requieren procesos de negocios flexibles que los soporten TI de manera efectiva en toda la organización. Ante este panorama, la problemática que se intenta resolver aquí es la siguiente: ¿cómo la $A E$ se puede gestionar mediante estrategias de TI? A fin de alcanzar el objetivo se utilizó el método Deslizar -ordenarse, rotular, integrar, priorizar-, el cual consistió primero en la organización de la información bibliográfica recuperada de bases de datos estructuradas, información que luego se etiquetó con la finalidad de clasificarla y priorizarla para su lectura y análisis. Se destaca como principal conclusión de este trabajo el hecho de que las organizaciones más abiertas al cambio son las que asumen el desafío de transformarse utilizando las TI, y en las que la AE es una aliada estratégica en ese cambio al gestionar de forma adecuada los procesos del negocio.

Palabras clave: arquitectura empresarial; gestión de tecnologías de información; cambio organizacional; estrategia organizacional; gestión del cambio; gestión organizacional. 


\section{Enterprise Architecture: a strategy for organizational change supported by information technology management. A bibliographic review}

\section{ABSTRACT}

The main objective of this article is to conduct a literature review on Enterprise Architecture (EA), the strategic management of Information Technology (IT), and organizational change, presenting their definitions and association in order to address the change in organizations. According to different studies in which EA is mentioned as an agile approach, we identified the main components of the organization and their relation with the purpose of achieving the business objectives. Currently, several companies rely on different strategies on account of their complexity and, therefore, require flexible business processes that support IT effectively throughout the organization. Given this panorama, the problem that we are trying to solve here is the following: How can EA be managed by means of IT strategies? In order to achieve this objective, the Slide method was used - sorting, labeling, integrating, and prioritizing - which first consisted of organizing the bibliographic information that was retrieved from structured databases. This information was then labeled in order to classify it and prioritize it for reading and analysis. The main conclusion of this work is that the organizations that have a more open attitude towards change are those that assume the challenge of transforming themselves using IT, and in which EA becomes a strategic ally by adequately managing business processes.

Keywords: enterprise architecture; information technology management; organizational change; organizational strategy; change management; organizational management 


\section{Arquitetura empresarial: estratégia para uma mudança organizacional apoiada no gerenciamento de tecnologias da informação. Uma revisão bibliográfica}

\section{RESUMO}

O objetivo principal deste artigo é realizar uma revisão bibliográfica a respeito da arquitetura empresarial (AE), do gerenciamento estratégico de tecnologias da informação (TI) e da mudança organizacional, apresentando sua definição e sua associação com o fim de enfrentar a mudança nas organizações. De acordo com diferentes estudos nos que se menciona a AE como um enfoque ágil, se identificam os principais componentes da organização e sua relação no propósito de obter os objetivos do negócio. Atualmente, várias empresas se apoiam em diferentes estratégias devido a sua complexidade e, portanto, requerem processos de negócios flexíveis suportados pelas TI de maneira efetiva em toda a organização. Ante este panorama, a problemática que se tenta resolver aqui é a seguinte: como a AE pode ser gerenciada mediante estratégias de TI? A fim de atingir o objetivo utilizou-se o método Deslizar - ordenar-se, etiquetar, integrar, priorizar -, o qual consistiu primeiro na organização da informação bibliográfica recuperada de bases de dados estruturadas, informação que depois se etiquetou com a finalidade da classificar e priorizar para sua leitura e análise. Destaca-se como principal conclusão deste trabalho o fato de que as organizações mais abertas à mudança sejam as que assumem o desafio de se transformar utilizando as TI, e onde a AE seja uma aliada estratégica nessa mudança ao gerenciar de forma adequada os processos do negócio.

Palavras-chave: arquitetura empresarial; gerenciamento de tecnologias de informação; mudança organizacional; estratégia organizacional; gerenciamento da mudança; gerenciamento organizacional. 


\section{Architecture entrepreneuriale: étude \\ bibliographique des stratégies de changement organisationnel à travers la gestion des technologies de l'information}

\section{RÉSUMÉ}

Cet article offre un passage en revue bibliographique, une définission et une association de l'architecture entrepreneuriale (AE), de la gestion stratégique des technologies de l'information (IT) et du changement organisationnel. Il identifie les différentes études dans lesquelles l'AE est considérée comme une approche efficiente, analyse les principales composantes de l'organisation et leurs relations avec l'obtention des objectifs commerciaux. Nombre d'entreprises s'appuient aujourd'hui sur des stratégies complexes et nécessitent des business plan flexibles et rapidement assimilables par le département informatique de l'organisation. Ayant pris en compte ces obligations sous-jacentes, nous tenterons de résoudre la problématique suivante: dans quelles mesures l'AE peut-elle être prise en charge par des stratégies informatiques? Pour y répondre, nous utiliserons la méthode Slide - ordonner, étiqueter, intégrer, hiérarchiser - consistant à organiser les informations bibliographiques extraites de bases de données structurées devant être étiquetées, classées et priorisées pour la lecture et l'analyse. La conclusion principale de cette recherche montre que les entreprises les plus ouvertes au changement sont celles qui relèvent le défi de la transformation digitale et dans lesquelles l'AE devient un allié stratégique permettant une gestion optimale de la conquête de marchés.

Mots clés: architecture d'entreprise; gestion de la technologie de l'information; changement organisationnel; stratégie organisationnelle; gestion du changement; gestion organisationnelle. 


\section{Introducción}

La teoría y la práctica de la arquitectura empresarial (AE) se ha desarrollado rápido en los últimos años. Por esta razón, la gran mayoría de empresas han iniciado su proceso de articulación con la AE mediante un cambio organizacional que se apoya con la gestión de tecnologías de la información (Gualteros, 2017; Millán et al., 2012). La arquitectura empresarial es un proceso de alineación entre una parte de la organización con las tecnologías de la información y las comunicaciones (TIC) (Rizal y Nanik-Qodarsih, 2018). La AE puede considerarse como aquella que integra los usos de las TIC en el interior de las organizaciones modernas, esto es, la gestión de la organización de manera holística (Arango-Serna, Londoño-Salazary BranchBedoya, 2015). Frente a esto, Arango-Serna et al. (2010) y Orantes et al. (2009) consideran que las organizaciones, con el fin de mantener altos niveles de competitividad, se enfrentan a un entorno de funcionamiento y operatividad que es complejo, cambiante y basado en un contexto de globalización, por tanto, las organizaciones deben estar en capacidad de hacer frente a esos cambios y adaptarse, para así obtener escenarios que les permitan mayores oportunidades a la hora de negociar.

La gestión del cambio organizacional es considerado por Rizal y NanikQodarsih (2018) un factor crítico de éxito en la implementación de la AE, pues al reconocer y gestionar el cambio permite que este se incorpore mejor a las tecnologías de la información (TI) y se expanda a los dominios de aplicación de la AE.

Ahora bien, las TI han estado presentes desde hace décadas y su uso ha sido cada vez más amplio, especialmente en organizaciones con ambientes turbulentos, esto es, organizaciones sometidas a cambios constantes del entorno (Bergeron, Raymond y Rivard, 2001). Ante los riesgos en el entorno se considera que las TI deberían ser más flexibles y los gerentes estar más alerta para adaptar los sistemas de información a dichos cambios externos (Bergeron et al., 2001). 
Al mismo tiempo, la gestión de TI debe estar orientada estratégicamente al cambio de la organización, en el entendido de que las TI permiten implementar estrategias comerciales, para que de esta manera la empresa adopte una postura competitiva más fuerte (Bergeron et al., 2001).

En consecuencia, la AE tiene como principal objetivo aplicarse a la manera de una práctica capaz de conducir al desarrollo de la empresa desde la perspectiva de alineación entre la estrategia y los objetivos de TI.

Sin embargo, el propósito de este documento es responder la siguiente pregunta orientadora: ¿cómo la arquitectura empresarial puede convertirse en una estrategia de cambio organizacional con ayuda de las tecnologías de la información?

En tal sentido, el objetivo de este trabajo es realizar una revisión bibliográfica acerca de la arquitectura empresarial, la gestión estratégica de tecnologías de la información y el cambio organizacional, presentando su definición y la asociación de estos a fin entender cómo la AE y las TI se combinan en una estrategia para afrontar el cambio en las organizaciones.

Este documento está estructurado en cuatro secciones: método, desarrollo del tema, discusión, y conclusiones. En la sección del método se explica la forma en la cual se realizó la revisión bibliográfica con un método sistemático. El desarrollo del tema contiene las definiciones de arquitectura empresarial, gestión estratégica de TI y cambio organizacional, así como las dimensiones de la arquitectura empresarial y las relaciones entre estas. La discusión incluye una aproximación a la respuesta de la pregunta orientadora. Para finalizar se exponen las conclusiones.

\section{Método}

El desarrollo de este documento de revisión sobre el tema de arquitectura empresarial se basó en el método descriptivo a través de revisión de literatura, utilizado por Arango-Serna, Londoño-Salazar y Branch-Bedoya (2015), Gualteros (2017), Millán et al. (2012) y Orantes, Gutiérrez y López (2009), en el cual se 
ubican diferentes referencias bibliográficas de bases de datos estructuradas como, por ejemplo, Scielo, Science Direct, IEEE Xplore y Google Académico. Para la búsqueda se utilizaron ecuaciones con los temas de AE, gestión estratégica de Tl y cambio organizacional, se definieron criterios de selección de los documentos y, finalmente, los artículos seleccionados profundizan en el tema con principal interés en su aplicabilidad en empresas.

\subsection{Intención de la revisión}

Buscar las definiciones de arquitectura empresarial, gestión estratégica de TI y cambio organizacional, así como la asociación entre estos términos para responder la pregunta de investigación. A fin de dar cumplimiento a la intención de la revisión se propone el siguiente diseño metodológico de trabajo en etapas de investigación.

\subsection{Definición de fuentes bibliográficas}

La búsqueda de la literatura para la elaboración del artículo de revisión se fundamentó en diferentes tipos de fuentes:

- Scielo. Es una colección de revistas científicas electrónicas, a texto completo de acceso libre y gratuito disponible en línea.

- Science Direct. Base de datos en línea que permite la búsqueda y la recuperación de textos completos.

- IEEE Explore. Base de datos en línea que permite la búsqueda y la recuperación de textos completos de publicaciones del Institute of Electrical and Electronics Engineers (IEEE) y el Observatorio EstratégicoTecnológico (OET).

- Google Académico. Buscador especializado en documentos académicos y científicos en el que se encuentran resúmenes y citas, entre otras fuentes de información. 
- Repositorios universitarios. Son espacios o archivos en los que se depositan de forma digital materiales derivados de la producción científica o académica de una institución. Entre estos se consideraron los repositorios de Universidad Nacional Abierta y a Distancia (UNAD), de la Universidad Santiago de Cali (USC) y de la Universidad Nacional de Colombia (UNAL), entre otros.

\subsection{Estrategia de la búsqueda}

Los descriptores utilizados para la búsqueda de las referencias bibliográficas fueron:

- arquitectura empresarial

- gestión estratégica de tecnologías de información

- arquitectura empresarial + gestión de TI

- cambio organizacional + TI

\subsection{Criterios de selección}

A partir de la lectura de los títulos de las publicaciones y su resumen se seleccionaron los artículos de acuerdo con los siguientes criterios:

- el documento da cumplimiento al objetivo de esta investigación

- el documento tiene información sobre la arquitectura empresarial, la gestión de las tecnologías de la información y el cambio organizacional

- el archivo está completo y puede descargarse

- el documento es único, no está duplicado

En la búsqueda en las bases de datos se leyeron los resúmenes y se procedió a realizar la lectura a profundidad para la fundamentación de este artículo. 


\subsection{Organización de la información}

Para la organización de la información se utilizó el método Deslizar ordenarse, rotular, integrar, priorizar- el cual ayudó a reducir la complejidad de análisis:

- Ordenarse. De las fuentes bibliográficas recolectadas se tomaron aquellas que presentaron mayor fundamentación teórica y se organizaron dependiendo de su objetivo principal, de tal manera que fuera posible clasificar fácilmente. A continuación, se listan los documentos seleccionados:

-«Arquitectura empresarial. Una visión general»;

-«A systematic literature review: critical success factors to implement enterprise architecture»;

-«Arquitecturas empresariales: gestión de procesos de negocios vs. arquitecturas orientadas a servicios ¿se relacionan?»;

-«Exploring the future of enterprise architecture: a Zachman perspective»;

-«Fundamentos de gestión de servicios TI basado en ITIL»;

-«Estudios de las prácticas de arquitectura empresarial en las grandes empresas del Valle del Cauca»;

-«Enfoque de arquitectura de solución, mecanismo para reducir la brecha entre la arquitectura empresarial y la implementación de soluciones tecnológicas»;

-«Model maintenance based in CO BIT for the applications architecture in TOGAF Enterprise Architectures-Momcae»;

-«Propuesta para la definición de la arquitectura empresarial»; 
-«Propuesta de una arquitectura empresarial para una institución de educación superior como un apoyo a su desarrollo frente a los retos del siglo XXI»;

- Rotular. Se clasificaron las publicaciones según las categorías de información propuestas para el artículo:

-definiciones;

-la arquitectura empresarial como estrategia de organización;

-dimensiones de la arquitectura empresarial.

- Integrar. Por medio de las categorías encontradas se realizó una revisión a fin de asociar cada una dentro del documento y se interpretó la relación entre la arquitectura empresarial como estrategia para un cambio organizacional apoyada con la gestión de tecnologías de la información.

- Priorizar. A partir del objetivo del artículo de revisión se tiene en cuenta la información según el nivel de importancia, la claridad del tema, el manejo y las conclusiones obtenidas del estudio.

\section{Desarrollo del tema}

La implementación de una arquitectura empresarial parte de un conjunto de directrices arquitectónicas que permitan asegurar un desarrollo armónico entre los modelos y las necesidades de la empresa con los procesos de negocio y las tecnologías de la información (Arango-Serna et al., 2010). En este sentido, las estrategias de TI deben partir de la misión, la organización y las tecnologías requeridas para el cambio y las necesidades de la empresa (Arango-Serna et al., 2010).

A fin de entender la noción de «arquitectura empresarial» es necesario explicar los términos que la componen por separado. Por una parte, arquitectura, según la IEEE Computer Society (Orantes et al., 2009), es la organización lógica de cualquier cosa o la organización fundamental de un 
sistema compuesto por sus componentes, la relación entre cada uno y con su medio ambiente, así como los principios que guían su diseño y evolución. Por otra, empresarial se refiere a cualquier colección de organizaciones que tiene un conjunto común de objetivos.

Es importante tener claro el origen del concepto arquitectura empresarial, el cual se origina en 1987 con la publicación del artículo de J. Zachman en el Diario IBM Systems, titulado «Un marco para la arquitectura de sistemas de información» (Arango-Serna et al., 2010; Gualteros, 2017; Lapalme et al., 2016). En ese documento Zachman establece la visión de la arquitectura empresarial y el desafío que representa al proponer un modelo para la arquitectura de sistemas de información (Arango-Serna et al., 2010; Gualteros, 2017; Orantes et al., 2009; Zachman, 1987). En esencia, el reto es administrar la creciente complejidad que representa el surgimiento de los sistemas de información, soportados en sistemas computacionales (Arango-Serna et al., 2010; Zachman, 1987), por lo que su propuesta es ahora una ontología para representaciones descriptivas de empresas (Orantes et al., 2009).

Según Zachman (1987), como se cita en Arango-Serna et al. (2010), «el éxito del negocio y los costos que ello conlleva dependen cada vez más de sus sistemas de información, los cuales requieren de un enfoque y una disciplina para la gestión de los mismos». Además, propuso un marco de trabajo enfocado en arquitectura empresarial -ZFEA por sus siglas en inglés-con dos ejes: uno vertical y otro horizontal. El eje vertical proporciona perspectivas de la arquitectura y el eje horizontal una clasificación de los diferentes artefactos de esta (Gualteros, 2017; Lapalme et al., 2016).

Otras definiciones sobresalientes de arquitectura empresarial se relacionan a continuación.

Ross, Well y Robertson (2006) definen la arquitectura empresarial como la lógica organizativa de los procesos de negocios y los activos de tecnología de información (Arango-Serna et al., 2010). 
En el libro Enterprise architecture at work, Lankhorst (2009) indica que la arquitectura empresarial es un conjunto coherente de principios, métodos y modelos utilizados en el diseño y la realización a nivel empresarial de la estructura organizacional, los procesos de negocio, los sistemas de información y la infraestructura (citado por Arango-Serna et al., 2010).

Fernández y Wijegunaratne (2010), por su parte, indican que la arquitectura empresarial se entiende como un mapa y una herramienta para la transformación y la evaluación organizacional (citados por Iglesias y Nieto, 2015).

Para Gualteros (2017), la arquitectura empresarial integra la gestión de una organización, la cual adopta una vista holística que abarca, entre otros aspectos, datos, información, procesos de negocio, sistemas de información e infraestructura tecnológica, y nace como una disciplina que evoluciona desde modelos administrativos y de gestión tales como la teoría organizacional y la teoría de sistemas.

En el mismo sentido, Orantes et al. (2009) expresan que una arquitectura empresarial bien estructurada le permite a la organización alcanzar un balance entre eficiencia tecnológica e innovación del negocio, pues su objetivo es proporcionar de manera ordenada un marco de trabajo preciso y propio que apoye las estrategias y las metas del negocio, además de que señalan los cuatro componentes de la arquitectura empresarial y cómo se relacionan, tal como se muestra en la figura 1.

Figura 1. Componentes básicos de las arquitecturas empresariales.

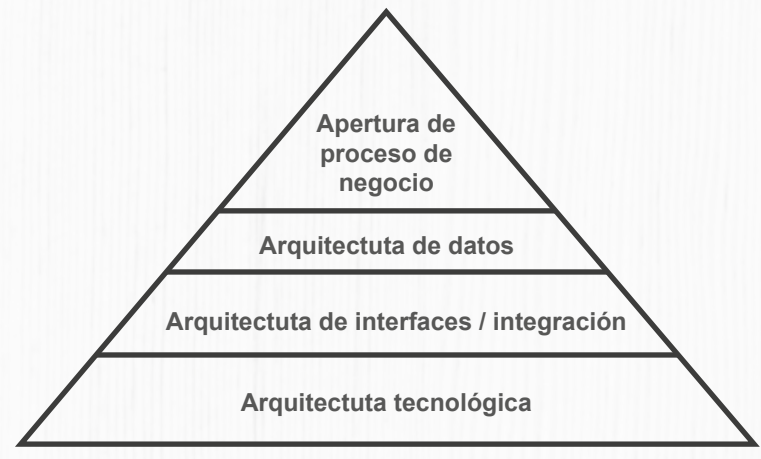

Fuente. Orantes et al., 2009. 
Ahora bien, en la AE se identifican seis componentes: estrategia, gobierno de TI, información, sistemas de información, servicios de tecnología, uso y apropiación. Su principal objetivo es garantizar la correcta alineación de la tecnología y los procesos de negocio en una organización, con el propósito de alcanzar el cumplimiento de los objetivos estratégicos (Martínez-Navarro, Jiménez-Toledo, Hernández-Pantoja, Benavides-Calvache y CarlosamaPantoja, 2017).

\subsection{Dimensiones de la arquitectura empresarial}

La descripción de la arquitectura empresarial se realiza con base en las dimensiones o los dominios asociados a las vistas arquitecturales que soportan los objetivos del negocio (Iglesias y Nieto, 2015). Estas dimensiones son cuatro y se enlistan en la tabla 1.

Tabla 1. Dimensiones AE.

\begin{tabular}{|c|c|c|}
\hline Dimensión & Descripción & Autores \\
\hline $\begin{array}{c}\text { Arquitectura de negocio } \\
\text { - procesos- }\end{array}$ & $\begin{array}{l}\text { Describe los flujos de la } \\
\text { información del negocio } \\
\text { y los intereses de los } \\
\text { usuarios del sistema, así } \\
\text { como sus componentes, } \\
\text { relaciones y objetivos. }\end{array}$ & $\begin{array}{c}\text { Gonzales-Inga y Tarifeño } \\
\text { Montero (2016); González- } \\
\text { Campo y Lozano-Oviedo } \\
\text { (2020); León-Bazan et al. } \\
\text { (2018); Rodríguez-Ríos } \\
\text { (2015); Schekkerman } \\
\text { (2008) }\end{array}$ \\
\hline $\begin{array}{l}\text { Arquitectura de } \\
\text { información } \\
\text { - datos- }\end{array}$ & $\begin{array}{c}\text { Maneja la estructura } \\
\text { de los datos y los } \\
\text { administradores de } \\
\text { bases de datos por medio } \\
\text { del estudio, el análisis, } \\
\text { la organización, la } \\
\text { disposición y la estructura } \\
\text { de la información. }\end{array}$ & $\begin{array}{c}\text { Arango-Serna et al. (2015); } \\
\text { Gonzales-Inga y Tarifeño } \\
\text { Montero (2016); González- } \\
\text { Campo y Lozano-Oviedo } \\
\text { (2020); León-Bazan et al. } \\
\text { (2018); Rodríguez-Ríos } \\
\text { (2015). }\end{array}$ \\
\hline
\end{tabular}




\begin{tabular}{|c|c|c|}
\hline $\begin{array}{c}\text { Arquitectura de } \\
\text { aplicaciones } \\
\text {-software- }\end{array}$ & $\begin{array}{c}\text { Se encarga de los } \\
\text { aspectos integrales del } \\
\text { proceso, los intereses y la } \\
\text { información del sistema } \\
\text { mediante la aplicación del } \\
\text { software. }\end{array}$ & $\begin{array}{c}\text { Aier, Riege y Winter, } \\
\text { (2008); Gonzales-Inga y } \\
\text { Tarifeño Montero (2016); } \\
\text { González-Campo y } \\
\text { Lozano-Oviedo (2020); } \\
\text { León-Bazan et al. (2018); } \\
\text { Rodríguez-Ríos (2015); } \\
\text { Wurman y Bradford } \\
\text { (1996). }\end{array}$ \\
\hline $\begin{array}{l}\text { Arquitectura de } \\
\text { tecnología } \\
\text { - hardware- }\end{array}$ & $\begin{array}{c}\text { Correlaciona los intereses } \\
\text { de estructura del } \\
\text { software y el hardware, } \\
\text { garantizando la } \\
\text { continuidad del negocio. }\end{array}$ & $\begin{array}{c}\text { Gonzales-Inga y } \\
\text { Tarifeño-Montero, (2016); } \\
\text { González-Campo y } \\
\text { Lozano-Oviedo, 2020; } \\
\text { León-Bazan et al., 2018; } \\
\text { Rodríguez-Ríos, 2015; } \\
\text { Schekkerman, (2008) }\end{array}$ \\
\hline
\end{tabular}

Fuente. Elaboración propia con base en Gonzales-Inga y Tarifeño-Montero, 2016; GonzálezCampo y Lozano-Oviedo, 2020; León-Bazan et al., 2018; Rodríguez-Ríos, 2015; y Schekkerman, 2008.

\subsection{Frameworks de la arquitectura empresarial}

La evolución de la arquitectura empresarial se enlaza con el desarrollo de diferentes marcos o frameworks (Lapalme et al., 2016). Estos marcos explican los componentes especiales que actúan como base para la estructuración y el ensamble en construcciones más complejas de la organización (ArangoSerna et al., 2010). En los estudios de Rizal y Nanik-Qodarsih (2018), ArangoSerna et al. (2010) y Ledesma-Alvear (2017) se relacionan los frameworks más utilizados:

- Federal Enterprise Architecture Framework (FEAF), EE. UU.;

- Zachman Framework for Enterprise Architecture (ZFEA);

- Extended Enterprise Architecture Framework (E2AF);

- The Open Group Architecture Framework (Togaf);

- Gartner Enterprise Architecture Framework (GEAF);

- Thailand Interoperability Framework;

- e-Gif;

- SAGA;

- COBIT; 
- DYA o Dynamic Architecture;

- Business-IT alignment (BITA);

- GC Enterprise Architecture and Standards (BTEP), Canadá;

- Ministerio de Tecnologias de la Información y la Comunicación (MinTIC);

- Department of Defense Architecture Framework (DoDAF).

Según Ledesma-Alvear (2017), se considera por criterios de importancia y por su adaptabilidad el framework Togaf, sin embargo, se debe definir un modelo que permita evidenciar la realidad de la organización, trabajando con los interesados y formulando preguntas para definir lo que realmente se quiere con la implementación de una AE.

\subsection{La importancia y los beneficios de la arquitectura empresarial}

La AE identifica los componentes principales de la organización y su relación con el propósito de conseguir los objetivos de negocio (Orantes et al., 2009).

Entre los beneficios que obtiene la organización al adoptar un modelo de AE, según Gualteros (2017), se encuentran:

- mejorar la eficiencia operacional de la empresa;

- identificar las inversiones más rentables a realizar;

- generar valor agregado para la empresa;

- alinear TI con los objetivos estratégicos de la empresa;

- establecer lineamientos y estándares transversales a toda la organización;

- contar con información confiable y de alta calidad para una mejor toma de decisiones;

- aprovechar de manera eficiente las oportunidades de negocio soportadas en las fortalezas de la empresa.

En el nivel de sistemas de información se tiene por cometido automatizar los procesos de negocio en cuestión, es decir, su propósito es dar el soporte de TI requerido por los medios establecidos a fin de lograr los fines estipulados (Arango-Serna et al., 2010). 


\subsection{Gestión estratégica de TI y el cambio organizacional}

Las empresas que perciben incertidumbre en su entorno luchan por sobrevivir y prosperar en mercados cada vez más dinámicos, inestables y competitivos, de manera que para los teóricos de la organización se ha asumido durante mucho tiempo que esta incertidumbre juega un papel importante en las relaciones entre tecnología y estructura en la organización (Bergeron et al., 2001). También se considera que el aumento de la inestabilidad en el entorno hace que la adquisición de información sea más continua, variante y de mayor alcance. En este sentido, la gestión de TI debe estar orientada estratégicamente al cambio de la organización, entendiendo que la TI permite implementar estrategias comerciales, para que de esta manera la empresa adopte una postura competitiva más fuerte (Bergeron et al., 2001).

Por otra parte, debido a la necesidad de adoptar nuevos procesos, objetivos y métodos, es probable que la organización requiera aplicar cambios organizacionales transcendentales. Un ejemplo de estos cambios necesarios ha sido la implementación de las tecnologías en las organizaciones, lo cual ha venido a representar nuevos métodos y procesos en su funcionamiento (Garbanzo-Vargas, 2015).

Si bien se ha escrito mucho sobre la importancia del ajuste entre la función de los sistemas de información y la estrategia organizacional, la perspectiva dominante considera que la TI desempeña un papel moderador (Bergeron et al., 2001).

Por su parte, la gestión de los servicios de TI dentro de una organización puede entregar valor a los clientes y esto puede lograrse con ITIL (information technology infrastructure library), un compendio de publicaciones o librerías que describen de manera sistemática un conjunto de «buenas prácticas» para la gestión de los servicios de TI (Gualteros, 2017; Orozco y Valencia, 2008).

Por su parte, Ayala (2008) afirma que para lograr el éxito en las empresas es necesario que las $\mathrm{TI}$ se encuentren alineadas con las estrategias de negocio y les brinden soporte. 


\section{Discusión}

Con base en la revisión anterior se puede obtener una primera aproximación a la respuesta de la pregunta orientadora ¿cómo la arquitectura empresarial puede convertirse en una estrategia de cambio organizacional con ayuda de las tecnologías de información? Ahora, si bien se encuentra relación entre los temas que se abordaron en este documento, se puede decir que el cambio en la organización generado por situaciones turbulentas del entorno permite que la organización se autogestione a través de la incorporación y uso de las TI, lo que la lleva a reformular su estrategia de negocio, y esto, a su vez, a obtener una visión integradora de la organización con base en la AE.

En síntesis, una arquitectura empresarial bien estructurada le permite a la organización alcanzar un balance lógico entre eficiencia tecnológica e innovación del negocio, y determinar qué secciones específicas de este pueden modernizarse con seguridad, en busca de ventajas competitivas.

La arquitectura empresarial ha evolucionado con el objeto de hacer frente a dos problemas importantes que se presentan de forma creciente en la gestión de TI. Zachman (1987) indica que el éxito del negocio y los costos que esto conlleva dependen cada vez más de sus sistemas de información, los cuales requieren de un enfoque y una disciplina para su gestión.

En razón a que el cambio organizacional puede llevar al éxito mediante el buen uso de la $\mathrm{AE}$, en estos momentos las organizaciones se encuentran en una adaptación en su estructura y en la manera de comunicarse, por lo cual se considera la necesidad de implementar dicho cambio a partir del costobeneficio y generar así una nueva oportunidad de crecimiento. Lo anterior, toda vez que el uso de TI genera más probabilidades de alcanzar procesos de innovación y una gestión organizacional competitiva. 


\section{Conclusiones}

Las empresas enfrentan un desafío tecnológico al gestionar los procesos de negocio, por lo cual deben contar con una herramienta o un conjunto de herramientas integradas que les permitan poner en práctica los nuevos retos a los que se enfrentan y así continuar en el mercado y seguir siendo competitivas, mientras brindan a sus clientes el servicio deseado.

El desarrollo de la arquitectura empresarial se debe entender como la descripción integral y estructurada de los diferentes elementos que conforman la empresa. La realizan equipos interdisciplinarios que conocen muy bien la organización, sus procesos, las líneas de negocio y la forma en que esta evoluciona; y, acogiéndose a las reglas y principios corporativos, aplican las técnicas y las metodologías establecidas, se arriesgan a proponer, a innovar y a disfrutar del proceso de construcción de diferentes procesos y proyectos que apoyan el desarrollo del negocio y tienen la capacidad de percibir, pensar y proyectar la empresa con una visión global e integral, sin perder de vista el contexto en el que esta se desenvuelve.

\section{Referencias}

Aier, S.; Riege, C.; Winter, R. (2008). Classification of enterprise architecture scenarios-an exploratory analysis. An International Journal, 3(1), 14-23. DOI: https://dx.doi.org/10.18417/emisa.3.1.2

Arango-Serna, M. D.; Londoño-Salazar, J. E.; Branch-Bedoya, J. W. (2015). Solution architecture approach, mechanism to reduce the gap between enterprise architecture and implementation of technological. Dyna, 82(193), 117-126. DOI: https://doi.org/10.15446/dyna.v82n193.46752

Arango-Serna, M. D.; Londoño-Salazar, J. E.; Zapata-Cortés, J. A. (2010). Arquitectura empresarial: una visión general. Revista Ingenierías Universidad de Medellin, 9(16), 101-111. 
Ayala, A. (8 de diciembre de 2008). ITIL, COBIT, CMMI: cómo integrar los estándares para un buen Gobierno de TI. Helkyn Coello Blog. Recuperado de https://bit.ly/2YvS7Y8

Bergeron, F.; Raymond, L.; Rivard, S. (2001). Fit in strategic information technology management research: an empirical comparison of perspectives. Omega, 29(2), 125-142. DOI: https://doi.org/10.1016/S03050483(00)00034-7

Garbanzo-Vargas, G. M. (2015). Desarrollo organizacional y los procesos de cambio en las instituciones educativas, un reto de la gestión de la educación. Revista Educación, 40(1), 67-87. DOI: https://doi.org/10.15517/ revedu.v40i1.22534

Gonzales-Inga, J. P.; Tarifeño-Montero, L. M. (2016). Análisis comparativo de frameworks de arquitectura empresarial para el alineamiento estratégico de tecnologías de información (Tesis de pregrado). Universidad Señor de Sipán, Perú. Recuperado de https://bit.ly/2FOxbFf

González-Campo, C. H.; Lozano-Oviedo, J. (2020). Propuesta para la definición de la arquitectura empresarial. Dimensión Empresarial, 18(1). DOI: $\underline{\text { https:// }}$ doi.org/10.15665/dem.v18i(1).2109

Gualteros, A. C. (2017). Modelo de arquitectura empresarial para empresas innovadoras en el sector de telecomunicaciones (TELCO) (Tesis de maestría). Universidad Nacional de Colombia, Colombia. Recuperado de https://bit.ly/2Ee7XQw

Iglesias, A.; Nieto, W. (2015). Un análisis de la adopción de programas de arquitectura empresarial en las organizaciones. Journal of Engineering and Technology, 4(1), 62-78.

Lapalme, J.; Gerber, A.; Van der Merwe, A.; Zachman, J.; De Vries, M.; Hinkelmannce, K. (2016). Exploring the future of enterprise architecture: a Zachman perspective. Computers in Industry, 79, 103-113. DOI: https:// doi.org/10.1016/j.compind.2015.06.010

Ledesma-Alvear, J. C. (2017). Frameworks de arquitectura empresarial (Tesis de Especialista). Universidad Nacional de La Plata. La Plata, Argentina. Recuperado de https://bit.ly/2YqmEqr 
León-Bazan, Y. Y.; Arana-Ureta, B. E.; Bonilla-Bermeo, J. D.; García-Plúa, J. C. (2018). Automatización e implementación de un sistema de administración de procesos de negocio (BPM) para el proceso de administración de proyectos basado en las metodologías Capability Maturity Model Integration (CMMI- DEV) nivel 3 para Servicios Profesionales. Espacios, 39(7), 19.

Martínez-Navarro, Á. A.; Jiménez-Toledo, R. A.; Hernández-Pantoja, G. A.; Benavides-Calvache, D. E.; Carlosama-Pantoja, J. A. (2017). Arquitectura empresarial como marco de trabajo para el desarrollo de software y la articulación de las dimensiones de bienestar universitario y las TI en la Universidad Mariana. 4(2), 165-167.

Millán, A. F.; Rodríguez, M. A.; González, E. M.; Alzate, J. W. (2012). Estudio de las prácticas de arquitectura empresarial en las grandes empresas del Valle del Cauca. Ingenium. 6(13), 45-54. DOI: https://doi.org/10.21774/ing. $\underline{\text { v6i } 13.87}$

Orantes, S.; Gutiérrez, A.; López, M. (2009). Arquitecturas empresariales: gestión de procesos de negocio vs. arquitecturas orientadas a servicios se relacionan. Tecnura, 13(25), 136-144. DOI: https://doi. org/10.14483/22487638.6676

Orozco, A. C.; Valencia, M. (2008). ITIL, un modelo para la gestión de servicios de $\mathrm{TI}$ en el contexto empresarial colombiano (Tesis de grado). Universidad Eafit, Colombia. Recuperado de https://bit.ly/3gl6BAg

Rizal, A.; Nanik-Qodarsih, B. S. (2018). A systematic literature review: critical success factors to implement enterprise architecture. Procedia Computer Science, 135, 43-51. DOI: https://doi.org/10.1016/j.procs.2018.08.148

Rodríguez-Ríos, C. Y. (2015). Qué es business process management (BPM). Definiciones y conceptos. Revista de La Escuela Colombiana de Ingeniería, (98), 23-29.

Schekkerman, J. (2008). Enterprise architecture good practices guide: how to manage the enterprise architecture practice. Bloomington: Trafford.

Wurman, R. S.; Bradford, P. (1996). Information architects. Nueva York: Graphis.

Zachman, J. (1987). A framework for information systems architecture. IBM Systems Journal, 26(3), 276-292. DOI: https://doi.org/10.1147/sj.263.0276 